\title{
IDENTIFICAÇÃO MICROQUIMICA DE MINERAIS PRIMÁRIOS DE POTÁSSIO NO SOLO
}

\author{
Renato A. Catani \\ Engenheiro agrônomo, Seç̧ão de Agrogeologia, Instituto Agronômico de Campinas
}

\section{1 - INTRODUÇÃO}

O conhecimento da composição mineralógica do solo constitui uma contribuição de valor, para esclarecer a natureza da riqueza potencial do solo, nos elementos de importância à nutrição vegetal. Além disso, a mineralogia do solo esclarece fenômenos relacionados com a gênese, físico-química e outros ramos da ciência do solo.

O presente trabalho é o resultado parcial de um estudo mineralógico referente a solos do Estado de São Paulo, executado pelo autor durante sua estada na Universidade de Missouri, nos Estados Unidos da América do Norte.

A finalidade dêste estudo é descrever uma técnica rápida para a identificação e contagem de minerais potássicos primários no solo.

\section{2 - O PROBLEMA}

Como é conhecido, os solos das regiões tropicais e subtropicais apresentam, em geral, uma riqueza potencial muito baixa nos elementos potássio, cálcio e magnésio. Êste fato é explicável pela decomposição muito rápida e intensa que se processa dos minerais primários daqueles elementos, nas condições climáticas apontadas.

Estudando a ocorrência do potássio nos solos do Estado de São Paulo, foi verificado que aquêle elemento se apresentava em outras formas, além da "trocável" (2). Posteriormente, foi determinado o teor total em potássio nos diferentes tipos de solos do Eistado de São Paulo e, dos resultados obtidos, chamou-se a atenção para os dos solos procedentes do Arenito Bauru, que são os seguintes :

NÚmERo DA AMOSTRA

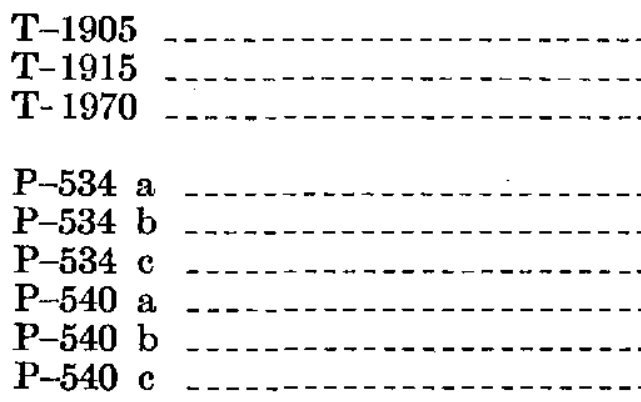

Teor trocável m. e. K/100 g de solo

0,28

0,20

0,32

0,16

0,08

0,45

0,30

0,33

0,19
T'eor total m. e. K/100 g de solo
5,20
6.30
15,80
15,40
18,00
16,00
24,00
36,40
37,20 


\section{(Continuaģão)}

Número da amostra

Teor trocável m. c. K/100 g de solo

P-547 a

$P .546$

$\mathrm{P}-54 \overline{6}$ c

$\mathrm{P}-295$ :

P-295 b

$\mathrm{P}-295$ c
0,31

0,31

0,30

0,09

0,08

0,01
Teor total

m. c. K/100 g de solo

6,20

6,30

5,80

6,60

5,60

7,10

Na primeira coluna estão representados os números das amostras de terras, onde $T$ designa amostra superficial, retirada de 0 a $30 \mathrm{~cm}$, e $P$, perfil. As letras $a, b$ e $c$ significam amostras tiradas de 0 a $40 \mathrm{~cm}, 40$ a $80 \mathrm{~cm}$ e a $150 \mathrm{~cm}$, respectivamente. Na segunda colına está o teor "trocável" extraído por solução de acetato de amônio normal com $\mathrm{pH}=7,0$. Na torreira coluna está representado o teor total extraído por meio de um ataque do solo eom ácido sulfúrico e ácido fluorídrico (5). As determinações do potásio foram executadas por fotometria de chama (3).

Conforme mostram êses dados, o potássio ocorre em outras formas além da "trocável" c cm percentagem relativamente elevada.

Para investigar as formas em que o potássio "não trocável" ocorre no solo procedente do Arenito Bauru, foram separadas divervas fraçôes, de acôrlo com o diâmetro das partículas do solo. Fm tôdas as frações foi determinario o teor total cm potássio e, em algumas, foi investigada a presença do minerais primários de potássio.

\section{3 - MATHRIAL W MTODOS}

() perfil 534 , com as camadas a e b, foi escolhido como material para o sutudo em questão porque é realmente um perfil típico de solo virgem, proredente do Arenito Bauru. Iocalizado na Estação Experimental de Pindorama, apresentava, ainda, mata primária e subidrófila.

\section{1 - SEPARACAO DAS MHFRSAS FRACOLS 100 SOLO}

A separação das diversas fraçocs do solo, de acôrdo com o diâmetro das partículas, foi feita por peneiras, para as fracoes de 2 a $0,250 \mathrm{~mm}$, do 0,250 a $0,105 \mathrm{~mm}$, e de 0,105 a $0,053 \mathrm{~mm}$, usando as peneirat de 60,150 e 270 malhas, respectivamente.

A. Eraçoes menores que $0,053 \mathrm{~mm}$ foram separadas por decantação, nwando-sé o métedo da pipeta a a fómula de Stokos (i) para calcular o tompo nocessário à sedimentação cias particulas. Os dados obtidos são os segrintes:

Frigions

$20,250 \mathrm{~mm}$ Camade a

(tamala b

$0,250 \div, 105 \mathrm{~mm}$

18.1

$0,1050,053 \mathrm{~mm}$

38,2

$2 !, 6$

$0,053-0,020 \mathrm{~mm}$

10,5

$0,020-3,010 \mathrm{~mm}$

0,7

$0,910 \cdot 0,002 \mathrm{mim!}$

0.8

suture ste 0,002 mor

7,1

17,2

39,8

21,7

13,1

0,7

0,3

5,0 
Como mostram êsses dados, a maior percentagem do material do solo está distribuída entre as frações de 2 a $0,250 \mathrm{~mm}$ até 0,053 a $0,020 \mathrm{~mm}$. A fração argila, isto é, menor que $0,002 \mathrm{~mm}$, também apresentou um teor que deve ser levado em conta.

\section{2 - TEOR EM POTÁSSIO DAS DIFERENTES FRAÇÕES}

Foi determinado o teor total em potássio das diversas frações, e os resultados obtidos são os do quadro $n .^{\circ} 1$.

Quadro 1.- Teores totajs, eln potássio, das camadas $a$ e $b$ do perfil de solo n. 534 , determinados em diversas frações, separadas segurdo o diâmetro das partículas

\begin{tabular}{|c|c|c|c|c|c|}
\hline \multirow{2}{*}{ Fração do solo analisada } & \multicolumn{2}{|c|}{ Camada $a$} & \multicolumn{3}{|c|}{ Camada $b$} \\
\hline & $\underset{\text { da fraçāo }}{\mathrm{K} \text { em } 100 \mathrm{~g}}$ & $\begin{array}{l}\text { K em } 100 \\
\text { de solo }\end{array}$ & $\underset{\text { da fração }}{\mathbf{K} \text { em }} 100 \mathrm{~g}$ & $\begin{array}{c}\mathrm{K} \text { em } 100 \\
\text { de solo }\end{array}$ & \\
\hline , & m. e. & m. e. & m. e. & m. e. & \\
\hline 0,250 a $0,105 \mathrm{~mm} \ldots$ & 14,8 & 5 & 15.3 & & 6,1 \\
\hline 0,105 a $0,053 \mathrm{~mm} \ldots$ & 18,4 & 4 & 16,3 & & 3,5 \\
\hline 0,053 a $0,020 \mathrm{~mm}$ & 30,9 & 3 & 29,8 & & 3,9 \\
\hline menor que $0,002 \mathrm{inm}$ & 17,4 & 1 & 21,3 & & 1,2 \\
\hline
\end{tabular}

Como mostram êsses dados referentes à distribuição do potássio nas diversas frações do solo, deve-se concluir que as frações de 0,25 a 0,105 $\mathrm{mm}$, de 0,105 a 0,053 e de 0,053 a $0,020 \mathrm{~mm}$ encerram minerais primários de potássio.

\section{3 - ESTLDO MICROQUIMICO DAS FIRAÇOES 0,250-0,105 mm, 0,105-0,053 $\mathrm{nm}$ e $0,053-0,020 \mathrm{~mm}$}

Já têm sido empregados métodos rápidos de identificação de minerais primários em sedimentos, conforme citam Marshall e Jeffries (7) e Krumbein e Pettijolın (6). Fm geral, as técnicas recomendadas são baseadas em reações microquímicas, que se desenvolvem na superfície dos mincrais após um tratamento determinado.

\subsection{1 - REMOÇÃO DOS ÓXIDOS IJE FERRO ADERENTES AOS GRANULOS DE SOLO}

Este tratamento foi feito com a finalidade de facilitar o exame microscópico do solo, pois a presença dos óxidos de ferro impedia o estudo pretendido.

O método empregado foi o de Jeffries (4), cujo resumo é o seguinte : Transferiu-se uma quantidade variável entre 0,5 a $2 \mathrm{~g}$ das frações a estudar, para um copo de $100 \mathrm{ml}$. Foram adicionados $40 \mathrm{ml}$ de solução a $10 \%$ de oxalato de potássio, agitoll-se e aqueceu-se a, aproximadamente, $80^{\circ} \mathrm{C}$ em banho-maria. Foram adicionados $10 \mathrm{ml}$ de uma solução de ácido oxálico a $9,5 \%$ c agitou-se. Deixon-se a temperatura elevar-se o máximo possivel, em banho-maria, e mergulhou-se uma fita de magnésio $(0,2 \mathrm{~g})$ elementar. 
Agitou-se, para homogenizar, e manteve-se a temperatura elevada por $\mathbf{5}$ minutos. Removeu-se a fita de magnésio, adicionaram-se $5 \mathrm{ml}$ da solução de ácido oxálico a 9,5\% e continuou-se a aquecer por mais 8-10 minutos. Transferiu-se para um tubo de centrífuga, centrifugou-se, lavou-se 3 a 4 vêzes com solução de $\mathrm{NaCl}$ a $5 \%, 3$ a 4 vêzes com solução de $\mathrm{HCl} 0,1 \mathrm{~N}$, 3 a 4 vêzes com água destilada e deixou-se secar. O material ficou, assim, pronto para ser preparado em lâminas.

\subsection{2 - PREPARO DAS LÂMINAS E CONTAGEM DOS MINERAIS (i)}

Em primeiro lugar deve-se preparar o meio fixador dos minerais. Esse meio, cuja finalidade é fixar os minerais em lâminas permanentes, para posteriores tratamentos químicos, é preparado a partir de um adesivo especial (weather stripping cement). Esste adesivo é usado nos Estados Unidos da América do Norte, na indústria de automóveis, e foi adaptado ao estudo de solos na Universidade de Missouri. No Estado de S. Paulo, a "Durex" Lixas e Fitas Adesivas Ltda. fabrica o produto denominado Durlok, que também se presta muito bem para a preparação do meio fixador.

Em um tubo de centrífuga, juntou-se 1 parte, em volume, do adesivo, a 2 partes, em volume, de acetato de amilo. Esta proporção não precisa ser exata. Agitou-se e centrifugou-se por 5-6 minutos para remover as partículas sólidas do adesivo. Preparado o meio fixador, a lâmina foi montada como segue :

a) Duas a três gotas do fixador foram adicionadas à lâmina de vidro e esperou-se adquirir uma consistência desejável. Em geral, de 10 a 20 minutos são suficientes.

b) Os minerais do solo foram distribuídos na superfície do fixador, de maneira que formassem uma camada uniforme. Evitou-se a formação de aglomerações, o que dificultaria o ataque e contagem dos grânulos. Esperaram-se 10 minutos.

c) As lâminas assim preparadas foram invertidas numa garrafa de solução de ácido fluorídrico (46-48\% de $\mathrm{HF}$ ), durante 5 minutos.

d) As lâminaś foram mergulhadas numa solução saturada de cobaltihexanitrito de sódio, durante 5 minutos. A solução de cobaltihexanitrito de sódio pode também ser preparada consoante já foi descrito (2), a partir de nitrato de cobalto, nitrito de sódio e ácido acético.

e) As lâminas foram lavadas em água destilada para eliminar o excesso de solução de cobaltihexanitrito de sódio e foram deixadas a secar.

f) Fêz-se a contagem dos minerais de potássio encontrados em 500 a 1000 grânulos, por meio de microscópio comum ou binocular. Pode ser usado com maior eficiência um microscópio "Kubiena", pois não só a iluminação é mais perfeita como também a contagem se torna mais fácil.

$\mathrm{O}$.tratamento descrito em $c$, isto é, o ataque com ácido fluorídrico, tem por finalidade expor o potássio do mineral para ulteriores reações

(1) A téenica de preparação das lâminas para o estudo de minerais primários no solo tem sido desenvolvida e aplicada por E. R. Graham e C. M. Woodruff, respectivamente, professor assistente e instrutor de solos, da Universidade de Missouri, Estados Unidos da América do Norte. 
químicas. Com o tratamento $d$, o potássio reage com o cobaltihexanitrito de sódio e orjgina um precipitado amarelo de cobaltihexanitrito de potássio e sódio, que reveste os grânulos. Desta mancira, os minerais que encerram potássio (os feldspatos potássicos) tornam-se amarelos, enquanto os outros não se alteram.

A contagem dos minerais foi feita com um aumento de 75 vêzes (10 vêzes na ocular o $7,5 \mathrm{em}$ objetiva) nas frações $0,25-0,105 \mathrm{~mm}$ e $0,105-0,053$ $\mathrm{mm}$. Para a fração $0,053-0,020 \mathrm{~mm}$ foi usado um aumento de 150 vêzes (20 vêzes na ocular e 7,5 na objetiva), mas, também, se pode usar o aumento de 75 vêzes já deserito.

$\Lambda$ iluminação foi feita com lâmpada comum, mas foi conduzida diretamente sôbre a lâmina.

\section{4 - RESUITADOS OBNIDOS}

Como foi dito anteriormente, os minerais primários potássicos adquirem uma côr amarolo-intensa, com o tratamento descrito. Os demais minerais, como quartzo, ilmenita, magnetíta, etc., não se alteram, tornando-se, portanto, fácil a distinção e contagem dos minerais potássicos. Executada a contagem, obtiveram-se os dados percentuais, conforme mostra a seguinte relação :

Frações do perfFl 534

Percentagem numprica de minerais motassicos

$\begin{array}{lrrr} & & \text { camada } & \text { camada } \\ 0,25-0,105 \mathrm{~mm} & 3,6 & 3,5 \\ 0,105-0,053 \mathrm{~mm} & \ldots \ldots & 4,6 & 4,6 \\ 0,053-0,020 \mathrm{~mm} & \ldots \ldots & 8,8 & 10,9\end{array}$

Os dados obtidos confirmam que o teor relativamente alto om potássio, nas amostras estudadas do solo procedente do Arenito Bauru, tem como responsável a presença de minerais primários de potássio. Tál fato é de singular importância, e esta é a primeira vez que foram constatados minerais de potássio no solo procedente do Arcnito Bauru.

Fstudos mineralógicos posteriores usando o microscópio e a técnica petrográfica revelaram que o mineral potássico presente nas amostras estudadas era o ortoclásio, KA1 $\mathrm{Si}_{3} \mathrm{O}_{8}$. Há, entretanto, necessidade de obter um número maior de dados para verificar a distribuição de minerais primários potássicos en outras áreas do mesmo solo.

\section{5 - CONCLCSOTES H RESUMO}

O presente trabalho trata do um estudo das formas de ocorrência do potássio no solo procedente do Arenito Bauru. Assim, foi relatada uma técnica microquímica rápida para a verificação e contagem de minerai: primários de potássio nas diversas frações do solo.

O problema que êste estudo procurou solucionar surgiu da seguinte maneira : algumas amostras do solo procedente do Arenito Bauru revelaram um tenr total om notássio relativamente alto. oue variou de 5.2 a 
$37,2 \mathrm{~m}$. e. de potássio por $100 \mathrm{~g}$ de solo. Os dados obtidos sugeriam a existência de outras formas de ocorrência do potássio, além da "trocável".

Das amostras analisadas, foi escolhido um perfil típico do solo procedente do Arenito Bauru, e que ainda estava coberto por mata primária.

Foram estudadas as camadas $a$ e $b$, dêsse perfil (de zero a $40 \mathrm{~cm}$ e de 40 a $80 \mathrm{~cm}$, respectivamente).

As diversas frações do solo, de acôrdo com o diâmetro das partículas, foram separadas e, depois de analisadas, demonstraram a presença de potássio em quantidades relativamente elevadas.

As lâminas, para estudo microquímico das diversas frações das camadas $a$ e $b$ do perfil analisado, foram preparadas com um adesivo especial, para fixação dos minerais. Tratamentos químicos subsequentes [ataque com $\mathrm{HF}$ e reação com $\mathrm{Na}_{3} \mathrm{Co}\left(\mathrm{NO}_{2}\right)_{6}$ ] permitiram a distinção entre os minerais potássicos e não potássicos.

Foram constatados minerais primários de potássio nas frações de 0,25 a $0,105 \mathrm{~mm}, 0,105$ a $0,053 \mathrm{~mm}$ e de 0,053 a $0,020 \mathrm{~mm}$. Foi feita uma contagem dos minerais, e os resultados obtidos indicaram que, nas amostras estudadas, os minerais primários de potássio são responsáveis pelo teor elevado naquele elemento.

\section{S U M M A R Y}

This paper reports a study, from a chemical and microchemical point of view, of the soil potassium in the type of soil originating from the Arenito Bauru.

A mechanical separation of the soil was earried out in order to study the potassium distribution in the soil. The fractions 0.250 to $0.105 \mathrm{~mm}, 0.105$ to $0.053 \mathrm{~mm}, 0.053$ to $0.020 \mathrm{~mm}, 0.020$ to $0.010 \mathrm{~mm}$ and 0.010 to $0.002 \mathrm{~mm}$ were isolated. The chemical data, i. e, the exchangeable and the total potassium entent pointed out the occurrence of primary minerals of potassium in the fractions, 0.250 to $0.105 \mathrm{~mm}, 0.105$ to $0.053 \mathrm{~mm}$ ind 0.053 to $0.020 \mathrm{~mm}$.

A simple microchemical technique was performed by which it was possible to differentiate the potassium bearing mineral from quartz, ilmenite, magnetite and other minerals.

Primary minerals of potassium were found in those fractions and this faet in licated that the total potassium eontent in the samples studied was due tol the presenes of those minerals.

\section{Literatura CITADA}

1. Baver, L. D. Soil Physies. pág. 31-34. J. Wiley and Sons. 1940.

2. Catani, R. A. A. Küpper. As formas "rocável" e "fixa" dos eations $\mathrm{K}^{+}, \mathrm{Ca}^{++}$e $\mathrm{Mg}^{++}$ros solos do Éstado do S. Paulo. Bragantia 9: 185-192. 1949 .

3. Catani, R. A. e J. E. de Paiva Neto. Dosagem do potássio e sódio pelo "fotômetro de chama" - Sua aplicação em anślise do solo. Bragantia 9: 175-183. 1949.

4. Jeffries, C. D. A rapid method of the removal of free iron oxides in soil prior to petrographic analysis. Soil Sci. Soe. Am. Proe. 11: 211-212. 1946.

5. Kolthoff, I. M. and Sandell, E. B. Text-book of quantitative inorganic analysis. pág. 752, Revised edition, The Macmillan Company, New York. 1945.

6. Krumbein, W. C. and F. J. Pettijohn. Manual of Sedimentary Petrography. pág. 495-497. Appleton-Century-Crofts, Ine. 1938.

7. Marshall, C. E. e C. D. Jeffries. Mineralogical methods in soil rezearch. Soil Sci Soc. Amer. Proc. 10: 397-405. 1945. 